\title{
Electrophysiological Evidence of Semantic Interference in Visual Search
}

\author{
Anna L. Telling, Sanjay Kumar, Antje S. Meyer, \\ and Glyn W. Humphreys
}

\begin{abstract}
Visual evoked responses were monitored while participants searched for a target (e.g., bird) in a four-object display that could include a semantically related distractor (e.g., fish). The occurrence of both the target and the semantically related distractor modulated the N2pc response to the search display: The N2pc amplitude was more pronounced when the target and the distrac-
\end{abstract}

\section{INTRODUCTION}

Visual search typically involves looking for a prespecified target among varying numbers of distractors. Current theories suggest that the target for search provides an attentional template held in working memory, which acts to bias attention toward relevant objects (e.g., Duncan \& Humphreys, 1989). Single-unit recordings in monkeys have provided evidence for the maintenance of search targets in working memory and for their influence on target selection. Chelazzi, Miller, Duncan, and Desimone (1993) reported that neurons tuned to features of a cued target in the inferior temporal lobe showed increased neural activity prior to search, consistent with the maintenance of a target template in working memory. Subsequently, the monkeys were required to make an eye movement to the target in a choice array of between two and five items. Prior to any saccade being initiated, the activity of neurons tuned to distractors was suppressed and the neurons tuned to the target remained active. This provides the neural basis for selecting a saccadic response to the target rather than to any distractor (see also Thompson, Hanes, Bichot, \& Schall, 1996).

Psychological evidence for the role of top-down knowledge in driving human visual search comes from studies showing that the efficient search for targets at the extreme of a stimulus dimension (e.g., for a large target relative to small and medium distractors) depends on foreknowledge of what the target is (Hodsoll \& Humphreys, 2001, 2005). Without this foreknowledge, search for targets at the extreme of their dimension becomes much less efficient.

University of Birmingham, UK tor appeared in the same visual field, and it was less pronounced when the target and the distractor were in opposite fields, relative to when the distractor was absent. Earlier components (P1, N1) did not show any differences in activity across the different distractor conditions. The data suggest that semantic distractors influence early stages of selecting stimuli in multielement displays.
In everyday life, many of the targets that we search for appear not among unrelated distractors, but rather among distractors that can be related to the object we are looking for-as when we search for a cup in a kitchen containing related objects such as saucers, jugs, and so forth. What are the consequences for search of having distractors that are related to the target being searched for? The first study to assess this was reported by Moores, Laiti, and Chelazzi (2003). In their Experiment 5, participants were asked to search for a known target (e.g., a motorbike). On some trials, a distractor was presented that was semantically related to the target for the search task (e.g., motorbike helmet). On trials where the target did not appear (targetabsent trials), accuracy was lower and reactions were slower when a related distractor was present rather than when all distractors were unrelated to the target. Eye movement measures also revealed effects of the related distractor, in this case, on target-present as well as target-absent trials. The presence of a related distractor reduced the likelihood of first saccades to the target (41\% vs. $47 \%$ when the distractors were unrelated), whereas on target-absent trials, more first saccades were directed to the related distractor than to any of the unrelated distractors (23\% vs. 17\%, respectively). Similar findings have since been obtained by Belke, Humphreys, Watson, Meyer, and Telling (2008): Distractors that were semantically related to the target interfered with search. This effect was independent of display size ( 4 vs. 8 items).

Because in these studies the semantic relation was between the distractor and the item being searched for, the data suggest either of two possibilities: (i) that activity from a template for the target can spread to semantically related items, or (ii) that the target's template is specified 
in semantic terms and there is sufficiently rapid extraction of the semantic properties of objects for both targets and distractors to activate the template, causing competition for selection from multiple locations. Because the effect of the semantic distractor is additive with display size, it appears that the semantic properties of distractors can attract attention in parallel for up to eight items. We return to consider these possibilities in the General Discussion.

\section{The Present Study}

In the present article, we used ERPs to examine the time course of the effect of semantic bias from a target template to related distractors. ERPs provide a fine-grained means of tracking the time course of visual selection because they give an on-line measure of when the variable of interest (in this case, the presence of a distractor semantically related to a target) influences processing. Our interest focuses on the N2pc, an enhanced negative deflection in the N2 time window, at posterior sites over the contralateral relative to the ipsilateral hemisphere coded with respect to the target position. The N2pc is visible at around 175-300 msec poststimulus onset and is thought to represent attentional selection (see Kiss, Van Velzen, \& Eimer, 2008). For example, the magnitude of the N2pc varies according to the difficulty of target selection (Luck \& Hillyard, 1994) and it covaries under the same conditions that determine the neural competition for selection observed in neurophysiological studies (Luck, Girelli, McDermott, \& Ford, 1997). We ask whether the presence of a semantic distractor is detected early enough in time to influence the N2pc amplitude.

Although the N2pc has not previously been studied in relation to search among semantic distractors, Eimer (1996) reported that this component was present when a target had to be discriminated from distractors on the basis of its semantic properties. In his Experiment 3, Eimer presented participants with two words in opposite locations either side of a fixation cross: one target and one distractor. The target words were either LINKS (left) or RECHTS (right) and the distractor words were WEISS (white) and BRAUN (brown). Participants responded according to the meaning of the target words, that is, pressing the left button if LINKS was present, and pressing the right button if RECHTS was present. A reliable N2pc (i.e., a larger negativity across the hemisphere contralateral to the target) was obtained over the posterior left hemisphere, suggesting that the N2pc can be sensitive to selection based on semantic properties of stimuli (see also Dell'Acqua, Pesciarelli, Jolicour, Eimer, \& Peressotti, 2007). Here we assess whether it is sensitive to the semantic relations between a distractor picture and the target being searched for in a multielement display.

In addition to the N2pc, we examined whether other ERP components were affected by the presence of a semantically related distractor. The P1 (first positive wave with an 80-130 msec peak after stimulus onset) and N1 (first negative wave with a 150-200 msec peak) components are typically thought to reflect differences in the early perceptual processing of stimuli (see Hillyard, Vogel, \& Luck, 1998; Luck \& Hillyard, 1995). By evaluating effects on these components, we assess whether there are differences in the early perceptual processing of displays when a related distractor is present. The P3 component occurs from 300 msec poststimulus onward and can reflect decisionmaking and response selection. For example, the P3 amplitude typically decreases during target-absent compared to target-present trials in search (e.g., Wolber \& Wascher, 2003; Luck \& Hillyard, 1990, 1994; for a review, see Kok, 2001). However, this component may, in addition, reflect the ease of target selection. For example, Wolber and Wascher (2003) reported decreasing amplitudes of the P3 component with increasing set sizes in conjunction search. Note that target selection is more difficult at larger set sizes as the difficulty of search increases.

In the present study, participants were presented with a word specifying the search target followed by a visual search array containing four objects. A picture matching the target word was present on half the trials. During these target-present trials, the picture of a semantically related distractor was either (i) presented in the same field as the target, (ii) presented in the opposite field to the target, or (iii) was absent from the display and replaced by an unrelated foil picture. During target-absent trials, a foil replaced the target and the semantic distractor was either (i) presented in the same field as the foil, (ii) in the opposite field to the foil, or (iii) it was absent. Figure 1 provides examples of the different conditions. Trials where both the target and the semantic distractor were present were separated according to whether these items were on the same or opposite sides of space because the ease of target selection may differ under these two conditions. In particular, selection may be easier when the target and semantic distractor are in the same field than when they are in opposite fields (when the stimuli may compete to determine whether attention should be shifted to the left or right visual field).

We expected to find slower responses when the target was absent rather than present, and slower responses when the distractor was present rather than absent (Moores et al., 2003). If semantic distractors influence early perceptual processing, changes in P1 and N1 amplitude would be expected. Effects on target selection may be expected on the N2pc component, whereas effects on decision-making may emerge on the P3 component.

\section{EXPERIMENT 1}

In some of the earlier studies of the effects of semantic distractors on search, participants were free to move their eyes (Experiment 5 in Moores et al., 2003; Experiment 1 in Belke et al., 2008). To avoid eye movement artifacts, participants in our EEG experiment (Experiment 2 below) were asked to maintain fixation at the center of the screen. Trials with eye movements were discarded. In Experiment 1, 
Target Present

A Distractor same side B Distractor opposite side C Distractor absent

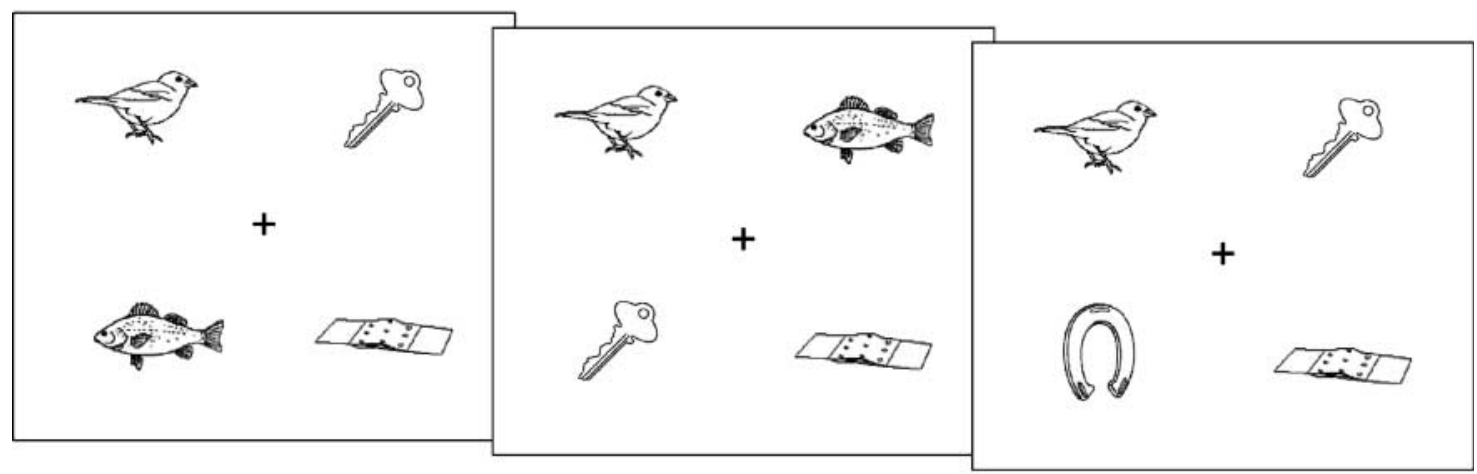

Target $=$ bird, Distractor $=$ fish, Distractor Foil $=$ horseshoe

Target Absent

D Distractor same side $\quad$ E Distractor opposite side $F \quad$ Distractor absent

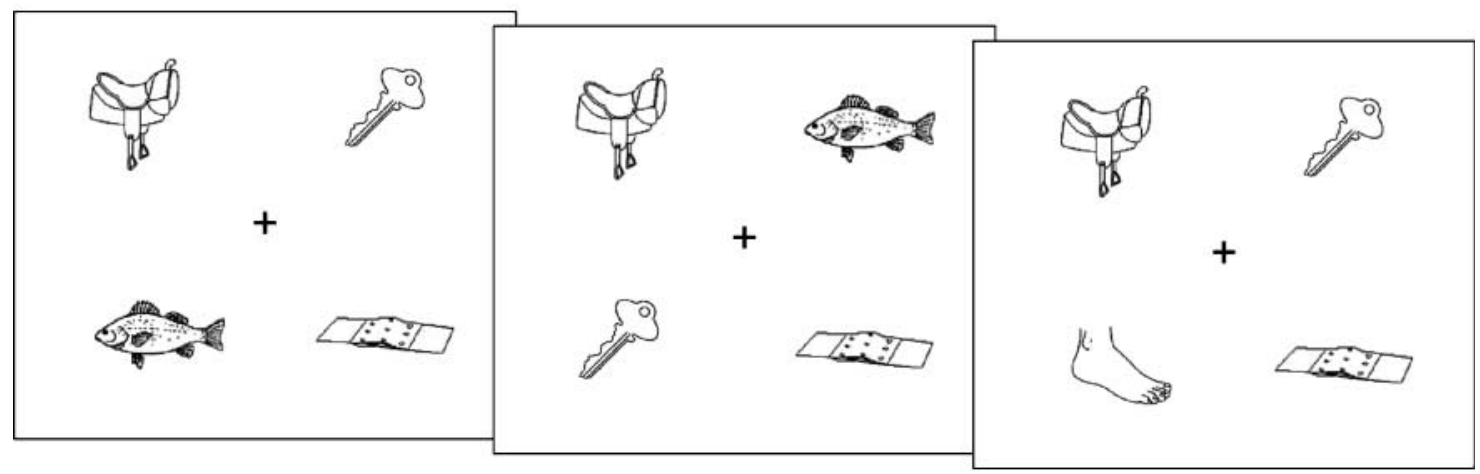

Target Foil $=$ saddle, Distractor $=$ fish, Distractor Foil $=$ foot

Figure 1. Sample displays used in each of the experimental conditions of Experiments 1 and 2 .

we only recorded eye movements and response speed but no EEG. The goal was to ensure that participants were able to carry out the search task and to determine whether a semantic effect on response speed and/or accuracy would be obtained, prior to running an ERP version of the experiment.

\section{Methods}

\section{Participants}

Twelve participants from the University of Birmingham were tested in return for course credits. They were aged between 18 and 26 years (mean age $=21$ years, 3 men). They reported their vision as normal or corrected to normal. All participants were right-handed. They were all native speakers of English.

\section{Stimuli}

The visual search display contained four objects presented at a distance of $11 \mathrm{~cm}\left(7.5^{\circ}\right.$ of visual angle from the partici- pant's position) diagonally from the center of each object to a central fixation cross. The objects fitted into frames of $7 \times 7 \mathrm{~cm}\left(5^{\circ}\right)$. The pictures were selected from Snodgrass and Vanderwart (1980) and a picture gallery provided by the Max-Planck-Institute for Psycholinguistics, Nijmegen (NL). All stimuli were black line drawings presented on a white background.

Participants were presented with a target word (e.g., bird) followed by a four-object search array. Each of the four objects was taken from a different set of 16 items: a target set, a distractor set, and two unrelated filler sets (see Appendix). On 50\% of trials, one of the objects was the target, for instance, a picture of a bird (a member of the target set). On the remaining trials, the target was replaced by foil (an unrelated object, randomly chosen from the target set), for instance, a picture of a saddle. Semantic distractor objects belonged to the same semantic category as the target (e.g., a picture of a fish). The semantic distractor occurred on 50\% of all target-present trials and $50 \%$ of all target-absent trials. On the remaining trials (the distractor-absent trials), the distractor was replaced 
by a foil unrelated to the target (an unrelated object, randomly chosen from the distractor set), for example, a picture of a horseshoe. The other two positions on the search display were taken up with objects from two unrelated filler sets.

The search items were chosen to minimize visual similarity between the targets and the related and unrelated distractors. In an independent rating study, in which the participants rated the visual similarity of the targetdistractor pairs on a 5 -point scale $(1=$ dissimilar, $5=$ identical), the average ratings for the two types of pairs were $1.6(S D=0.4)$ and $1.7(S D=0.6)$, respectively. This difference was not significant $(p>.05$, Wilcoxon test). A counterbalancing procedure ensured that each item appeared as both a related and unrelated distractor, eliminating effects due to the specific items.

Objects were arranged so that the target (or its foil) and the distractor (or its foil) appeared on the same field or opposite field to one another. The objects were positioned in all permitted locations on the screen (target topright, distractor bottom-left is an example of an opposite condition). There were four possible positions on the display for each factor of field (same or opposite), target status (present or absent), and distractor condition (present or absent). Each participant was exposed equally often (16 times) to each object from the target set when it was a target (during target-present trials) and when it was a foil (during target-absent trials). Likewise, each participant was exposed equally often (16 times) to each object from the distractor set when it was semantically related to the target (during distractor-present trials) and when it was unrelated (during distractor-absent trials). Across 16 items, this created 512 trials per participant.

Note that for the analyses of distractor absent trials, the field of the distractor foil in relation to the target or its foil is irrelevant. Therefore, the same and opposite field conditions were merged to create distractor-absent trials. The conditions were labeled according to target status (present or absent) and distractor condition (distractor on the same field as target/foil, opposite field as target/foil, or absent), as shown in Figure 1.

\section{Procedure}

The experimental stimuli were presented on a ViewSonic color monitor, $80 \mathrm{~cm}$ from the participant, set at a screen resolution of 1024 by 768 pixels. An SMI Eye Tracker (iView X, v1.6 Build 37) recorded eye movements at a sampling rate of one sample every $20 \mathrm{msec}$. First, participants familiarized themselves with a picture booklet containing pictures with names written beneath them for all stimuli used in the experiment and read the instructions from the computer screen. Next, the participants positioned themselves so that their chin was on a chin rest, which restricted head movements. The eye tracker was calibrated before the experiment began. Participants were asked to look at eight different points on the screen, presented in a random order. The points were an average of $7^{\circ}$ away from the center of the screen, and they were the same distance from the edge of the screen. The experimenter monitored eye movements throughout the experiment. Drift correction was carried out between trials, where necessary. Participants were forewarned of the possibility of this occurring as part of their instructions.

Each experimental trial began with a fixation cross in the center of the screen for $500 \mathrm{msec}$, followed by the target word, which lasted for $1 \mathrm{sec}$. Next, a fixation cross was presented for $600 \mathrm{msec}$. This was followed by the search display, with the fixation cross remaining in the center, which lasted until a response was given or up to $4.5 \mathrm{sec}$. The next trial began $1 \mathrm{sec}$ after response onset or $5.5 \mathrm{sec}$ after the onset of the display.

Participants were asked to search for the target picture among four pictures on the screen. Half of the participants were instructed to respond by pressing " $\mathrm{z}$ " on the keyboard if the target was present and " $m$ " if it was absent with their corresponding left and right index fingers. This was reversed for the remaining participants. Participants were instructed to respond as quickly and accurately as possible and to fixate the center of the screen throughout the trial. They were informed that any trials where they moved their eyes would be repeated at the end of the test block.

Each experimental block consisted of 64 trials, and each participant completed 8 blocks in total (512 trials). The order of the blocks was counterbalanced across participants. Each block contained an equal number of trials for each condition. A different random order of trials was used for each participant. Opportunities for breaks were provided between blocks.

For the eye movement analyses, a $4.2 \mathrm{~cm} \times 4.2 \mathrm{~cm}\left(3^{\circ}\right)$ interest area around the central fixation point was defined. Trials were categorized as eye error trials if participants fixated positions outside of this area for more than 600 msec. Such trials were repeated at the end of the experiment. Trials where eye tracking was not possible for more than $75 \%$ of the trial duration because the participant closed his or her eyes or looked away from the screen were likewise excluded and repeated. Each trial was repeated only once. The experiment lasted approximately $1 \mathrm{hr} 15 \mathrm{~min}$.

\section{Results and Discussion}

Eye errors consisted of 1.6\% missing samples and 14\% eye movement error (where an eye movement took place during the trial). The remaining trials were selected for further analysis. The first five trials of the first block were excluded as practice trials $(0.7 \%)$. Next, any responses with latencies that were outside of three standard deviations from the participant's mean reaction time (RT) were excluded (1.7\%). 
Table 1 presents the mean RT for correct responses and the accuracy data. The mean RT observed across distractor-present conditions (same and opposite) was 700 msec, whereas the mean RT in the distractor-absent conditions was $682 \mathrm{msec}$, suggesting a disruptive influence of the semantic distractor. Participant means were compared using a repeated measures analysis of variance (ANOVA) with the factors target status (present, absent) and distractor (same, opposite, or absent). There was a main effect of target status $[F(1,11)=34.78, p<.001$, $\left.\eta^{2}=.76\right]$, with faster RTs in the target-present than in the target-absent condition, and a borderline effect of distractor condition $\left[F(2,22)=3.38, p=.060, \eta^{2}=.24\right]$. There was no interaction $[F(2,22)=0.30]$. Planned comparisons showed a significant difference between the conditions where the distractor was on the same side as the target and where it was absent [an 18-msec difference: $t(12)=2.95, p=.013, \eta^{2}=.44$ ] only.

The corresponding analysis of the accuracy data revealed no differences between target-present and target-absent trials $[F(1,11)=3.88]$, but a significant difference between the distractor conditions $\left[F(2,22)=7.61, p=.017, \eta^{2}=\right.$ .41]. Planned comparisons showed significant differences between all distractor conditions [same side as target/foil vs. opposite side: $t(12)=2.78, p=.018, \eta^{2}=.41$; same side as target/foil vs. absent: $t(12)=2.21, p=.049, \eta^{2}=$ .31; opposite side to target/foil vs. absent: $t(12)=3.21, p=$ $.008, \eta^{2}=.48$ ]. Participants made significantly more correct responses when the semantically related distractor was either on the same side as the target (on target-present trials) or its foil (on target-absent trials) relative to when the related distractor was absent, whereas more errors relative to the last mentioned condition occurred when the related distractor fell on the opposite side of space to the target/foil.

This experiment confirms the effect of the semantic distractor reported by Moores et al. (2003), particularly on accuracy rates, but under conditions where eye movements were not permitted. However, the effects on RTs were less pronounced (only approaching significance). These same display conditions were used in Experiment 2, where EEG recordings were also taken.

\section{EXPERIMENT 2}

\section{Methods}

\section{Participants}

Eighteen participants from the University of Birmingham were tested in return for course credits or cash. They were aged between 18 and 26 years (mean age $=21$ years, 5 men). They reported their vision as normal or corrected to normal. All but one of the participants were righthanded. They were all native speakers of English. All participants provided informed consent and confirmed that they had not consumed alcohol or recreational drugs in the 24 hours prior to testing and that they were not on any prescriptive medications that may affect cognitive processes.

\section{Stimuli}

The experimental stimuli used in Experiment 2 were identical to those used in Experiment 1.

\section{Procedure}

The experimental procedure was identical to that in Experiment 1, with the following exceptions. The participants sat $75 \mathrm{~cm}$ from an SVGA color monitor with the keyboard on their lap. No chin rest was used. No trials were repeated on occasions where eye movements occurred. The participants were asked to keep as still as possible during the blocks, keeping eye movements, swallowing, and blinking to a minimum.

\section{EEG Recording and Analysis}

EEG was recorded continuously with $\mathrm{Ag} / \mathrm{AgCl}$ electrodes from 128 scalp electrode locations. The electrodes were placed according to the 10-5 electrode system (Oostenveld \& Praamstra, 2001) using a nylon electrode cap. Horizontal and vertical eye movements were monitored by unipolar electrodes placed at the outer canthus and infraorbital area of the left eye, respectively. Additional electrodes were used for references and ground. EEG and EOG signals were

Table 1. Results of Experiment 1: Mean Accuracy Rates and RTs for Each Experimental Condition with Standard Deviations in Parentheses

\begin{tabular}{lllr}
\hline Target Status & \multicolumn{1}{c}{ Distractor } & Accuracy (\% Correct) & RT (msec) \\
\hline Target present & Present, same side & $91(4)$ & $653(114)$ \\
& Present, opposite side & $82(12)$ & $653(114)$ \\
& Absent & $84(8)$ & $640(110)$ \\
Target absent & Present, same side & $90(6)$ & $750(132)$ \\
& Present, opposite side & $71(23)$ & $745(130)$ \\
& Absent & $85(13)$ & $725(129)$ \\
\hline
\end{tabular}


amplified by BioSemi Active-Two amplifiers and sampled at $1024 \mathrm{~Hz}$. The continuous EEG recordings were off-line referenced to the average of the left and right mastoids and band-pass filtered between 0.01 and $35 \mathrm{~Hz}$. Continuous EEG signals were segmented into epochs from $200 \mathrm{msec}$ before trial onset to $1100 \mathrm{msec}$ after trial onset for each of the conditions for each participant. Epochs were rejected if the voltage in horizontal eye electrodes exceeded $\pm 40 \mu \mathrm{V}$ and $\pm 100 \mu \mathrm{V}$ in any other electrodes. Five participants were excluded from further analysis due to excessive horizontal eye movements and/or excessive artifacts in the EEG data. For the remaining 13 participants, $7 \%$ of the data $(S D=7 \%)$ were rejected from further analysis because activities on horizontal eye movement channels exceeded $\pm 40 \mu \mathrm{V}$ and on other channels activities exceeded $\pm 100 \mu \mathrm{V}$. This resulted in a minimum of 40 trials per condition being used for ERP calculation. Prestimulus activity, occurring $200 \mathrm{msec}$ prior to search array onset, was used as baseline, and activities reported here were baseline corrected.

\section{Results}

\section{Behavioral Results}

The first five trials of the first block were excluded as practice trials $(0.7 \%)$. Next, any responses with latencies that were more than three standard deviations from a participant's mean RT were excluded (1.6\%). Table 2 presents the means for the accuracy and RT data observed in each condition of Experiment 2. Statistical analysis was carried out as for Experiment 1.

Accuracy rates showed no effect of distractor, only of target status $\left[F(1,12)=20.14, p=.001, \eta^{2}=.63\right]$. The mean accuracy across participants was 93\% for target-present trials and 96\% for target-absent trials.

$R T s$ on correct response trials showed a main effect of target status $\left[F(1,12)=54.92, p<.001, \eta^{2}=.82\right]$, with responses being $81 \mathrm{msec}$ slower on target-absent than target-present trials. There was also a main effect of distractor $\left[F(2,24)=11.85, p=.001, \eta^{2}=.50\right]$. Planned comparisons between the distractor conditions showed that RTs were significantly longer for trials where the related distractor was on the same side of fixation as the target/foil relative to when it was absent [a 30-msec dif- ference: $\left.t(12)=5.88, p<.001, \eta^{2}=.74\right]$, and RTs were also longer on trials where the related distractor was on the opposite side of fixation to the target/foil, compared with when it was absent [an 18-msec difference: $t(12)=$ 2.98, $\left.p=.012, \eta^{2}=.43\right]$. There was no interaction $[F(2$, 24) $=1.81]$.

\section{Electrophysiological Results}

Only correct response trials were included in the EEG analyses. The main component studied in this experiment was the $N 2 p c$. This component was analyzed at five pooled posterior and lateral occipital electrodes (PPO5h/ PPO6h, PPO3h/PPO4h, PO5h/PO6h, PO3h/PO4h, and PO7/O8) where maximal N2pc activity was observed across the conditions. Figure 2, Section 1, shows the current source density map (spline interpolation) of N2pc activity for target-present and target-absent conditions, grand-averaged across participants. N2pc maps were plotted from the difference waveforms of ipsilateral processing subtracted from contralateral processing. The resultant map is plotted from co-interpolation of voltage values between the scalp electrodes (see Lorenzo-Lopez, Amenedo, \& Cadaveira, 2008). N2pc amplitude was computed as the difference between contralateral scalp electrode activity and ipsilateral activity. Activity for the distractor conditions on target-present trials was calculated according to the position of the target (related distractor on the same side as the target, on the opposite side to the target, or absent). Activity for the distractor conditions on target-absent trials was calculated according to the position of the foil (related distractor on the same side as the foil, on the opposite side to the foil, or absent).

Note that the foils were randomly drawn from the set of items used as targets. This means that, although these items were unrelated to the target that was cued on the trial, they could have been targets for search on earlier trials. Hence, the foils could still exert some degree of attentional attraction on target-absent trials. By contrasting measurements of the N2pc relative to the location of the target (on target-present trials) and measurements of the $\mathrm{N} 2 \mathrm{pc}$ relative to the location of the foil (on target-absent

Table 2. Results of Experiment 2: Mean Accuracy Rates and RTs for Each Experimental Condition with Standard Deviations in Parentheses

\begin{tabular}{llcl}
\hline Target Status & \multicolumn{1}{c}{ Distractor } & Accuracy (\% Correct) & RT (msec) \\
\hline Target present & Present, same side & $93(4)$ & $675(94)$ \\
& Present, opposite side & $92(5)$ & $668(91)$ \\
Target absent & Absent & $93(3)$ & $761(92)$ \\
& Present, same side & $96(6)$ & $770(107)$ \\
& Present, opposite side & $97(3)$ & $753(110)$ \\
& Absent & $97(3)$ & $724(92)$ \\
\hline
\end{tabular}




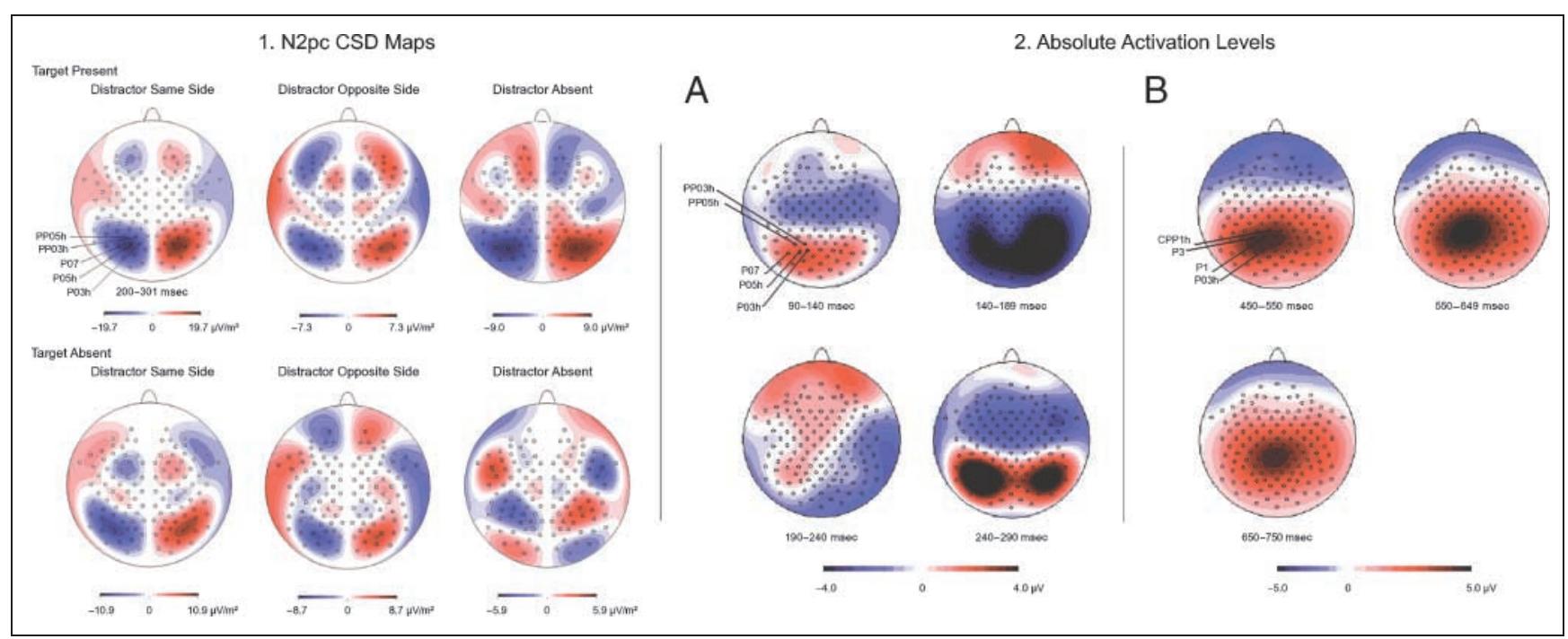

Figure 2. The left side of the figure, Section 1, shows current source density (CSD) distribution maps of the target present and absent conditions, with attention-related lateralized activities in the posterior region in the 200-300 msec time window (N2pc). N2pc maps are plotted from the difference waveforms of ipsilateral processing subtracted from contralateral processing. The resultant map is plotted from cointerpolation of voltage values between the scalp electrodes. The right side of the figure, Section 2, shows topographic maps of absolute activation levels for the target-present, distractor-present (same field) condition taken from the grand average across all participants and partitioned into 50 msec time windows. The first series of maps (A) shows occipital and posterior activity during the P1 (90-130 msec), N1 (155-195 msec), N2 (255-295 msec) time periods. The second series of maps (B) represent central occipital and posterior activity during the P3 (450-750 msec) time period. A similar pattern was found for the remaining five conditions.

trials), the analysis for target-absent trials paralleled that for target-present trials. On trials where the semantically related distractor was absent, we expected the N2pc to be larger when the target was present (target present, no distractor) than when the target was absent. This would reflect the increased potency of a current target (when present) relative to that of a former target (the foil). Our main interest, though, was how these N2pc effects were modulated by the presence of a distractor that was semantically related to the search target (although never a target itself). The N2pc, in both cases, should increase when the distractor fell on the same side as the target/foil, if the semantic distractor also attracts attention to that side. When the distractor fell in the opposite field to the target/foil, however, any N2pc to the target/foil may decrease due to competition for selection from the distractor in the other field.

Figure 1 shows sample displays for target present and absent trials. In A to $\mathrm{C}$ the target is on the left, so the contralateral electrode is located on the right side of the participant's head, and the ipsilateral electrode on the left side. Here, activity at the left electrode was subtracted from the activity at the right electrode, across all distractor conditions. For trials where the target was on the right, the calculation was reversed. In $\mathrm{D}$ to $\mathrm{F}$ the target was absent and was replaced by a foil. The position of the foil was used as a basis for classifying the laterality of electrodes: Here contralateral electrodes to the foil were on the right and ipsilateral electrodes on the left. Dividing the results in this way provides a baseline (when the target was absent) to measure neural changes when the target was present.
Visual inspection of the waveforms for the electrodes in the different conditions showed that the N2pc occurred between 200 and $300 \mathrm{msec}$ (see Figure 3). The mean amplitude $(\mu \mathrm{V})$ of the N2pc across this time window was examined for each condition at pooled electrode sites PO3h/PO4h, PO5h/PO6h, PPO5h/PPO6h, and PO7/O8 (as previous analyses showed no interaction between electrode site and distractor condition). Differences in target status (target present vs. target absent) and distractor condition (same, opposite, or absent) were assessed using repeated measures ANOVAs.

To qualify as an N2pc, the difference between activity on electrodes contralateral to the target and electrodes ipsilateral to the target should exceed $-0.3 \mu \mathrm{V}$ (see Woodman \& Luck, 2003). Figures 3 and 4 show that, according to this standard, the only occasion where no N2pc was elicited was on trials where both the target and the related distractor were absent $(0.17 \mu \mathrm{V})$. The most prominent N2pc was elicited on target-present trials when the semantic distractor was in the same field as the target $(-1.72 \mu \mathrm{V})$.

An ANOVA was conducted using the amplitude of the N2pc, with the factors being target status (target present vs. absent) and distractor condition (same, opposite, or absent). This showed reliable main effects of target status $[F(1$, 12) $\left.=11.76, p=.005, \eta^{2}=.50\right]$ and distractor condition $\left[F(2,24)=4.74, p=.035, \eta^{2}=.28\right]$. The N2pc was larger on target-present than target-absent trials (a difference of $-0.65 \mu \mathrm{V})$. There was no interaction between target status and distractor condition $[F(2,24)=3.08, p>.05]$.

The main effect of distractor condition was decomposed by comparing (i) same side with opposite, (ii) same side 
Figure 3. The top group waveforms display activity at the pooled electrodes contralateral and ipsilateral to the target or foil, grand-averaged across participants. The bottom group waveforms present differences between contralateral and ipsilateral processing. Larger contralateral negativity in the $200-300 \mathrm{msec}$ time window (N2pc) is observed for the target-present conditions (left). The time course of activity is indicated on the $x$-axis and the amplitude of electrode activity $(\mu \mathrm{V})$ on the $y$-axis. The negative is plotted upward, and that stimulus onset was at 0 msec. For display purposes, waveforms shown were $15 \mathrm{~Hz}$ high cutoff filtered.

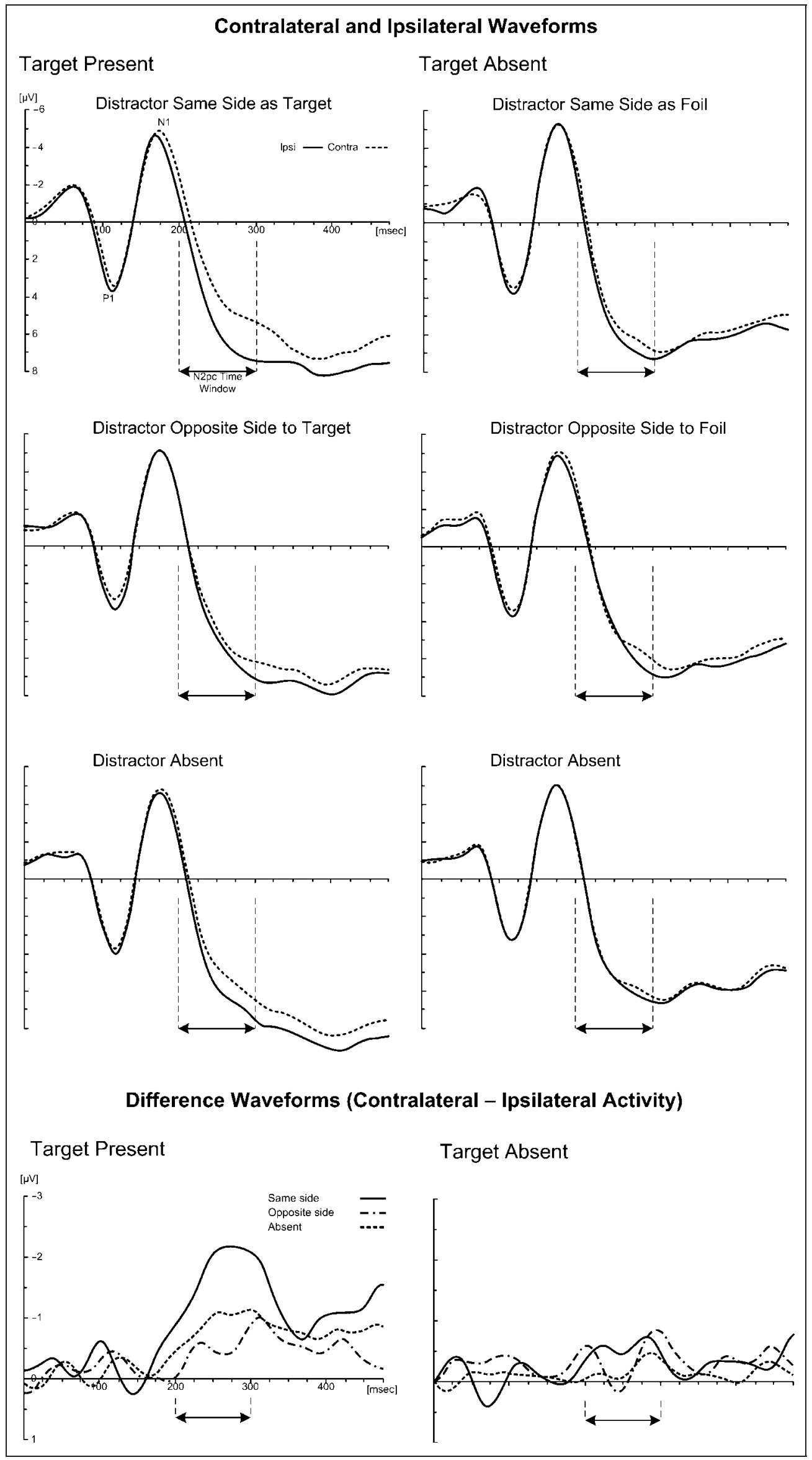


Figure 4. Mean N2pc activity across pooled electrodes PO3h/ PO4h, PO5h/PO6h, PPO5h/ PPO6h, and PO7/O8. For the condition where the semantic distractor fell on the same side as the target/foil, the N2pc was the same when it was measured relative to the target/foil and when it was measured relative to the distractor (because these items fell on the same side). When the distractor was absent, an $\mathrm{N} 2 \mathrm{pc}$ in relation to the distractor could not be measured. However, when the target/foil and the semantic distractor fell on opposite sides, the N2pc could be measured relative to either stimulus. Dark bars: N2pc measured relative to the target/foil; white bars: N2pc measured relative to the semantic distractor.

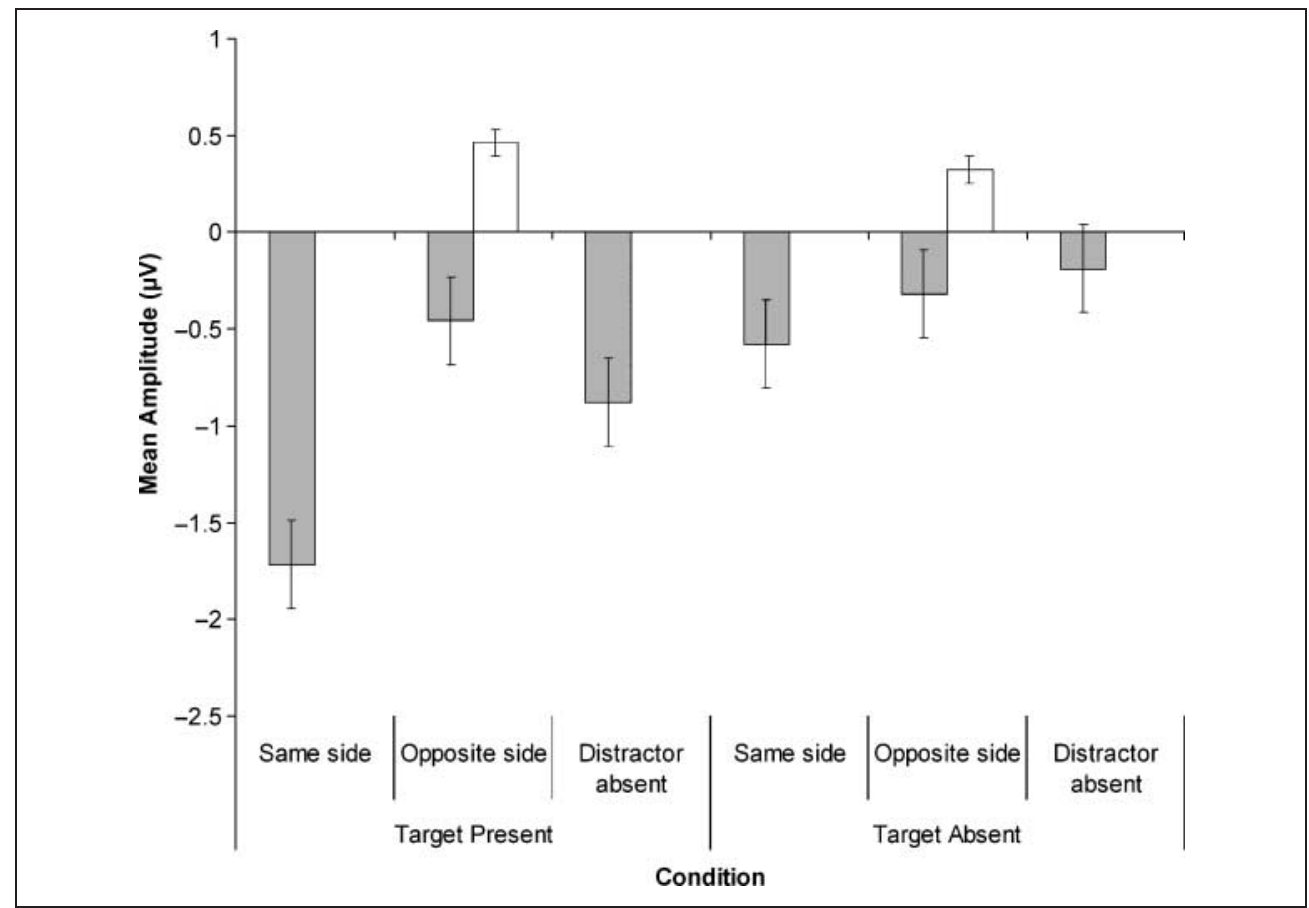

with absent, and (iii) opposite with absent distractor conditions, averaged across target-present and target-absent trials. Planned comparisons showed that the N2pc was greater when the related distractor was in the same field as the target/foil compared with when (i) it was in the opposite field $\left[t(12)=2.23, p=.046, \eta^{2}=.29\right.$; a difference of $0.76 \mu \mathrm{V}]$, and (ii) the related distractor was absent $\left[t(12)=3.14, p=.009, \eta^{2}=.45\right.$; a difference of $\left.0.62 \mu \mathrm{V}\right]$. The contrast between the conditions where the related distractor was opposite to the target/foil and the condition where it was absent was not significant $[t(12)=0.62, p=$ $.549, \eta^{2}=.03$; a difference of $\left.0.14 \mu \mathrm{V}\right]$. Finally, it should be noted that there was a significant difference between trials where the target was present and the related distractor was absent (the target-alone baseline; $-0.88 \mu \mathrm{V}$ ), and trials when the target was absent and the related distractor was present on the same side as the foil [the distractor-alone baseline; $\left.-0.58 \mu \mathrm{V} ; t(12)=2.33, p=.038, \eta^{2}=.31\right]$. This suggests that the distractor, when alone, did not generate as large an N2pc effect as when the target appeared without the related distractor. The foil alone did not generate an N2pc effect.

To evaluate the possibility that residual horizontal EOG (HEOG) activity contaminated the N2pc, we conducted further analyses on a bipolar HEOG channel constructed from electrode locations closest to the eyes (i.e., locations F9 and F10). Activity was quantified in the N2pc time window (200-300 msec). Analysis did not reveal a difference between conditions with the distractor in the same visual field compared to when it was in the opposite field to the target (the strongest contrast in the N2pc results). This was shown separately for targets on the left and targets on the right [left-side targets: distractor same vs. opposite, mean $=-0.95 \mu \mathrm{V}, t(12)=-0.929, p=.371, \eta^{2}=.067$; right-side targets: distractor same vs. opposite, mean = $\left.-1.01 \mu \mathrm{V}, t(12)=-0.913, p=.379, \eta^{2}=.065\right]$. The same analysis was carried out for target-absent trials according to the position of the foil. There was no effect of whether the distractor fell on the same or opposite side to the foil, when the foil fell in the left field [mean $=-0.87 \mu \mathrm{V}, t(12)=$ $-1.38, p=.193, \eta^{2}=.137$ ] or when it fell in the right field $\left[\right.$ mean $\left.=0.47 \mu \mathrm{V}, t(12)=0.839, p=.418, \eta^{2}=.055\right]$.

Taken together, the analyses indicate that the N2pc was larger for the target than for the foil, indicating that the current target exerted a strong effect on selection than items that had earlier been targets (the foils). In both cases, presenting a distractor that was semantically related to the target on the same side of space as the target/foil increased the N2pc further, whereas presenting the semantic distractor in opposite fields led to there being an overall N2pc in relation to the target/foil. A final point to note is that, if, on trials where the distractor fell in the opposite field to the target/foil, the N2pc was measured not relative to the position of the target/foil but relative to the location of the distractor, then the direction of the effect reversed to a positive waveform (see Figure 4). We return to this point in the General Discussion.

Prominent P1 (110 msec) and N1 (175 msec) component peaks were visible on the electrodes studied for the N2pc component (i.e., PO3h/PO4h, PO5h/PO6h, PPO5h/PPO6h, and $\mathrm{PO} 7 / \mathrm{O} 8$ ) as shown in the Figure 3 waveform displays and Figure 2 topographic map set (A).

The peak amplitudes in the 90-130 msec time window were analyzed for the P1 using within-participants ANOVAs, with the factors of target status, and distractor condition. The data were collapsed across electrode field (contralateral 
Table 3. P1 and N1 Pooled Mean Peak Amplitude ( $\mu \mathrm{V})$

\begin{tabular}{llcc}
\hline Target Status & \multicolumn{1}{c}{ Distractor } & P1 & N1 \\
\hline Target present & Present, same side & 5.20 & -7.31 \\
& Present, opposite side & 4.55 & -7.40 \\
& Absent & 5.00 & -6.71 \\
Target absent & Present, same side & 4.67 & -6.98 \\
& Present, opposite side & 5.01 & -7.57 \\
& Absent & 4.48 & -7.11 \\
\hline
\end{tabular}

or ipsilateral to the target or foil) and pooled across electrode sites. (Analysis including electrode field as a factor did not reveal any interactions with this factor.) No main effects or interactions occurred for any of the measures [largest $F(1,12)=2.28, p>.05$ ].

The same analyses were carried out for the N1 component (time window 155-195 msec). Again, no significant main effects or interactions were found [largest $F(1,12)=2.16$, $p>$.05]. The presence of a target or a semantic distractor had no effect on the P1 or the N1 (see Table 3 for the means for both components).

Finally, the $P 3$ component was analyzed. Isopotential contour maps for each condition indicated a bilateral centralposterior activity for the P3 component. The period of positive deflection began at around $450 \mathrm{msec}$ and shifted toward a negative deflection after $750 \mathrm{msec}$. Electrodes were selected for pooling according to which electrodes had the largest and most consistent positive deflections for all conditions: CPP1h, P1, P3, and PO3h (left); CPP2h, P2, P4, and PO4h (right), as seen in Figure 2, Set B. Due to the lack of a defined peak, only the mean amplitude in the shifting $100 \mathrm{msec}$ time window starting from 450 to $750 \mathrm{msec}$ was analyzed. A within-participants ANOVA was conducted with the factors target status (present/ absent), distractor condition (same, opposite, absent), and time window (450-550, 551-650, 651-750 msec). Target-present trials generated a larger $\mathrm{P} 3$ response than target-absent trials [an effect of $2.35 \mu \mathrm{V} ; F(1,12)=37.98$, $\left.p<.001, \eta^{2}=.76\right]$. There was also an interaction between time window and target status only $[F(2,24)=13.62, p=$ $\left..002, \eta^{2}=.53\right]$. There was a trend for the $\mathrm{P} 3$ to be greater on trials where the related distractor was absent than on trials where the related distractor was present (on either the same side or on the opposite side of fixation to the target/foil), although this failed to reach significance $[F(1$, $\left.12)=2.78, p=.087, \eta^{2}=.19\right]$. This effect was larger on target-present trials, although it tended to emerge at the longer time windows when the target was absent (see Figure 5).

\section{GENERAL DISCUSSION}

This study examined the time course of semantic interference in visual search. The behavioral data show that the presence of a distractor that was semantically related to the target slowed reactions and it also tended to increase the error rates (particularly when the distractor was on the opposite side of space to the target; Experiment 1). These findings are similar to those obtained by Moores et al. (2003, Experiment 5). The EEG analysis further demonstrated that the effects of the related distractor emerged at around $200 \mathrm{msec}$ and influenced the magnitude and onset latency of the N2pc component. This confirmed that the presence of a semantic distractor was detected early enough in time to influence the N2pc. The N2pc amplitude was greater when the related distractor fell on the same side of space as the target (or the foil, on target-absent trials) than when it either fell on the opposite side of space or was absent. The N2pc amplitude, measured relative to the
Figure 5. Mean $\mathrm{P} 3$ activity across pooled electrodes CPP1h/CPP2h, P1/P2, P3/P4, and $\mathrm{PO} 3 \mathrm{~h} / \mathrm{PO} 4 \mathrm{~h}$, divided between the three time windows.

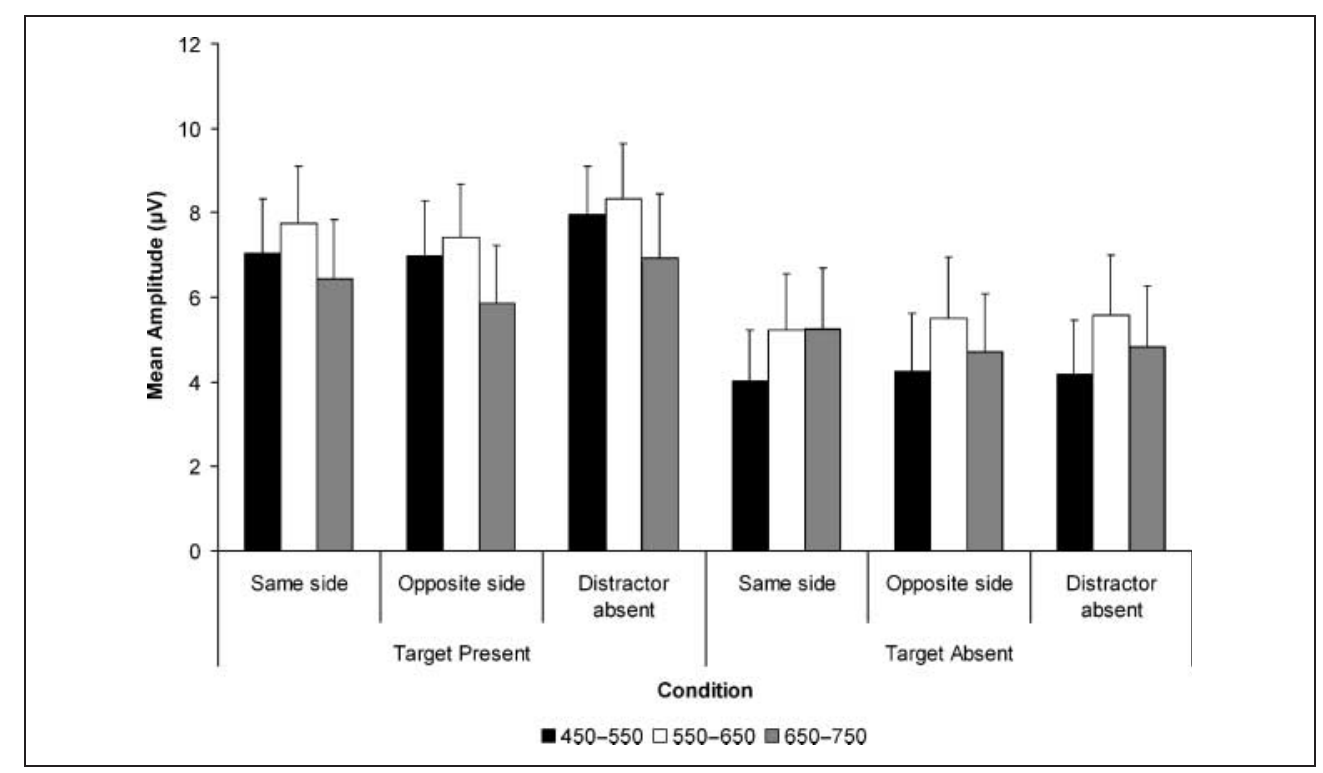


location of the target or its replacement foil, tended to be smallest when the distractor fell on the opposite side of space to the critical stimulus. This confirmed that the N2pc is sensitive to semantic relations between a distractor picture and the target being searched for in a multielement display. These results support Eimer's (1996) finding that the N2pc can be influenced by relatively high-level properties of a display (see also Eimer \& Kiss, 2007; Kiss et al., 2007).

In general, the N2pc amplitude was greater on trials where the target was present relative to when it was absent. Given that target-absent trials yielded longer RTs, this result is consistent with the N2pc reflecting the ease of selecting the target rather than the difficulty of search per se. Previous studies indicate that the N2pc may reflect the attentional effects found in area V4 and the inferotemporal cortex of monkeys (see Hopfinger, Luck, \& Hillyard, 2004), with the N2pc amplitude increasing when the allocation of attention to one hemifield increases. This was the case when the target is present, and this effect of the target was further modulated by the presence of a semantically related distractor. Our data suggest that attention is allocated to one hemifield more easily when both the target and the related distractor are there, as shown by increased $\mathrm{N} 2 \mathrm{pc}$ amplitude, compared with when the related distractor is either absent or on the opposite side of space. However, a greater N2pc was elicited by a target, in the absence of a related distractor, than by a related distractor in the absence of the target. This indicates that there was appropriate task-based selection of the target.

Although the N2pc amplitude was greater for trials where the related distractor appeared on the same side of space as the target, compared with trials where the related distractor was absent, RTs were slower. This suggests a discrepancy between the time taken to select a side of space and the time subsequently taken to select the target on the side initially selected. One way to think of this is as follows. Having the related distractor on the same side of space as the target facilitates the initial selection of that side, but the presence of the related distractor along with the target then slows target selection. This is not surprising, given that a related distractor will activate the target template, hence, create competition in the decision about whether the target is present or absent (cf. Bundesen, 1990). However, when the related distractor is on the same side as the target, there is additional evidence for stimuli on that side matching the template, leading to a stronger spatial bias in selection. The $\mathrm{N} 2 \mathrm{pc}$ amplitude appears to represent this bias. We term this the hierarchical account of selection; there is first selection of the side of space and then selection of the target within the selected side.

An alternative account for our findings is that the N2pc amplitude is the algebraic sum of two independent selection processes, one in response to the target and the other in response to the related distractor. The absence of an interaction between target status and distractor condition supports the summing of two selection processes. When the target/foil and the related distractor appear on the same side of space, their effects sum to create a stronger effect. Interestingly, if the N2pc is measured relative to the location of the distractor, rather than the target/foil, the N2pc reverses (becoming positive) on trials where the distractor and target/foil fall in opposite fields. Hickey, Di Lollo, and McDonald (2008) have recently noted the ambiguity of N2pc activity in conditions where the target and the distractor are lateralized, because in this case, there may be contributions to any overall N2pc from both items. The independent contributions of target and distractor items may be extracted by comparing N2pc activity when only one of the two items is lateralized and the other is placed in the vertical meridian. Using this approach, Hickey et al. (2008) isolated an early contralateral ERP component, which they associated with distractor suppression, the distractor positivity $\left(\mathrm{P}_{\mathrm{D}}\right)$. This was separated from the ERP component associated with target processing, the target negativity $\left(\mathrm{N}_{\mathrm{T}}\right)$. Hickey et al. concluded that the resulting N2pc was the sum of these two components, each contributing to the attentional selection of a target. The reversed (positive) deflections found in the present study, when waveforms were analyzed in relation to the position of the distractor, is consistent with a process of distractor suppression taking place. The important point, though, is that the presence of the semantic distractor influenced the stage of stimulus selection reflected in the N2pc.

The earliest components measured in this experiment, P1 and N1, showed no differences in activity according to the presence of the related distractor or even the target (on target-present vs. target-absent trials). Luck and Hillyard (1994) reported differences in both the P1 and N1 components on target-present versus target-absent trials in a search task where targets could be discriminated from distractors by a simple feature. However, the present results indicate that, with complex pictorial displays, the differences between targets and distractors are not sufficient to influence these early ERP components. Instead, the data suggest that there needs to be more protracted processing of the displays (taking around $200 \mathrm{msec}$ ) for differences between the pictures to be utilized.

The P3 may reflect aspects of target selection along with decision processes (Nieuwenhuis, Aston-Jones, \& Cohen, 2005; Desmedt, 1981). Our results showed that P3 activity from 450 to 750 msec decreased during target-absent trials, in line with the $\mathrm{P} 3$ reflecting either the ease of target selection or the ease of making the final decision (target-present decisions being easier than absent; see Chun \& Wolfe, 1996). In addition, conditions where the distractor was present tended to have decreased activity compared to when the distractor was absent, consistent with target selection/ decision-making being easier when the semantic distractor was not competing with the target. The magnitude of any P3 effect was not sensitive to whether the related distractor appeared on the same or the opposite side of space relative to the target, indicating that, at this stage in processing, the influence of the distractor occurred independently of the side of space it fell on. 


\section{The Visual Search Process}

The present results suggest the following analysis of the process of searching for a target picture among pictures of distractor objects. The analysis of the early ERP components (P1 and N1) suggest that, with the current stimuli, the initial $200 \mathrm{msec}$ or so of visual analysis proceeds in a manner that is relatively immune to the definition of an item as a target or distractor and also to whether any distractor is semantically related to the target for the search task. Following this, enough information is extracted from the items in the display to enable contact to be made with a template for the target and for target selection to begin. This process is influenced by the presence of distractors that are semantically related to the target. We can conceptualize interference from a related distractor occurring in at least two ways. On one view there is a spread of activity from the template held for the target to templates for related items. These other templates may be activated by the related distractor, when present, creating competition between templates for selection. An alternative is that the template for the target is created in relatively abstract semantic terms so that it is activated by distractors that are semantically related to the target (e.g., containing similar semantic features). This activation of a semantic template then leads to one stimulus being selected and this must be subsequently verified in order for participants to make the correct response. There is some recent evidence suggesting that search may be directed by relatively abstract, semantic information held in working memory. Soto and Humphreys (2007) asked participants to hold in mind a verbal label (e.g., red triangle) while they performed a visual search task. The search items (oriented lines) could appear within different shapes, one of which could correspond to the stimulus held in memory (the red triangle). Search times were strongly affected by the match between the verbal label and the search display, even though the memory stimulus was irrelevant to the search task. Soto and Humphreys proposed that there was relatively rapid semantic processing of search displays, which allowed extracted features to be matched to a semantically specified template for the target. The same may hold here. Indeed, we note that the target in the present experiments was defined by a verbal label rather than an image, which may encourage participants to use a relatively abstract template to guide search. It would be of interest to examine whether similar effects would arise if the target was defined as being a particular picture-effects based on matching information from the search display to a semantic template might decrease under this circumstance.

Our failure to find effects of the presence of either the target or the related distractor within the first $200 \mathrm{msec}$ or so after display onset stands at odds with recent work suggesting that at least some types of stimuli can make rapid contact with stored knowledge to direct search pro- cesses. For example, Bacon-Mace, Kirchner, Fabre-Thorpe, and Thorpe (2007) have recently shown that there can be selection of a saccade to an animate target on one side of space when displays are exposed for just $40 \mathrm{msec}$ under backward masking conditions. There are several differences between our experiment and theirs: They used full color images of natural scenes, the targets were animate objects, and only two stimuli were presented per trial. It may be that the processing of animate objects, supported by more fully specified visual images, is sufficiently rapid to lead to selection within a shorter time period than that revealed here. Interestingly, Belke et al. (2008) used the same paradigm as that employed here, tracking participants' free eye movements rather than ERPs, and found that the effects of the semantic distractor were equally large when displays of eight rather than four items were presented. This suggests that there is parallel extraction of the semantic features that may drive the current effects. Hence, the reason why we failed to find evidence for selective responses to targets and related distractors within 200 msec was not that critical features cannot be extracted in parallel, but apparently, any parallel processing needs to operate across this time period before target selection emerges.

Finally, at later stages of processing, effects of target presence/absence arise (on the $\mathrm{P} 3$ component). The magnitude of the P3 may reflect the difficulty of the final decision process along with the ease of target selection. There were minimal effects on the P3 of whether related distractors were on the same or opposite side to targets. This suggests that, if there is a component of the P3 influenced by the related distractor, this does not reflect the selection of one side of space, but rather the decision whether any selected item is the target.

In sum, the main finding of this article is that, in visual search for a target in a multielement array, the semantic properties of the stimuli are retrieved early enough to influence the N2pc (at around $200 \mathrm{msec}$ ). There is early semantic competition for selection in search.

\section{APPENDIX: MATERIALS}

\begin{tabular}{lcll}
\hline Target & Related Distractor & Target & Related Distractor \\
\hline arrow & bullet & organ & tuba \\
bird & feather & plane & ship \\
cigarette & ashtray & racket & bat \\
comb & brush & saddle & horseshoe \\
crown & scepter & shirt & trousers \\
hammer & drill & thread & rope \\
hand & foot & screw & hook \\
lock & key & & \\
nose & eye & & \\
\hline
\end{tabular}


Fillers: Set 1: torch, swan, tie, lollypop, pear, bell, cloud, football, card, mouse, plaster, flower, weight, belt, butterfly, broom. Set 2: finger, button, propeller, feather, king, face, horse, church, nail, hair, key, shuttlecock, ashtray, nut, needle, bow.

\section{Acknowledgments}

This work was supported by grants from the BBSRC, ESRC, MRC (UK), and the EU.

Reprint requests should be sent to Anna L. Telling or Glyn W. Humphreys, School of Psychology, The University of Birmingham, Edgbaston, Birmingham, B15 2TT, UK, or via e-mail: annatelling@ gmail.com; g.w.humphreys@bham.ac.uk.

\section{REFERENCES}

Bacon-Mace, N., Kirchner, H., Fabre-Thorpe, M., \& Thorpe, S. J. (2007). Effects of task requirements on rapid natural scene processing: From common sensory encoding to distinct decision mechanisms. Journal of Experimental Psychology: Human Perception and Performance, 33, 1013-1026.

Belke, E., Humphreys, G. W., Watson, D. G., Meyer, A. S., \& Telling, A. L. (2008). Top-down effects of semantic knowledge in visual search are modulated by cognitive but not perceptual load. Perception \& Psychophysics, 70, 1444-1458.

Bundesen, C. (1990). A theory of visual-attention. Psychological Review, 97, 523-547.

Chelazzi, L., Miller, E. K., Duncan, J., \& Desimone, R. (1993). A neural basis for visual-search in inferior temporal cortex. Nature, 363, 345-347.

Chun, M. M., \& Wolfe, J. M. (1996). Just say no: How are visual searches terminated when there is no target present? Cognitive Psychology, 30, 39-78.

Dell'Acqua, R., Pesciarelli, F., Jolicour, P., Eimer, M., \& Peressotti, F. (2007). The interdependence of spatial attention and lexical access as revealed by early asymmetries in occipito-parietal ERP activity. Psychophysiology, 44, 436-443.

Desmedt, J. E. (1981). P300 in serial tasks: An essential post-decision closure mechanism. Progress in Brain Research, 54, 682-686.

Duncan, J., \& Humphreys, G. W. (1989). Visual-search and stimulus similarity. Psychological Review, 96, 433-458.

Eimer, M. (1996). The N2pc component as an indicator of attentional selectivity. Electroencephalography and Clinical Neurophysiology, 99, 225-234.

Eimer, M., \& Kiss, M. (2007). Attentional capture by task-irrelevant fearful faces is revealed by the N2pc component. Biological Psychology, 74, 108-112.

Hickey, C., Di Lollo, V., \& McDonald, J. J. (2008). Electrophysiological indices of target and distractor processing in visual search. Journal of Cognitive Neuroscience, 21, 760-775.

Hillyard, S. A., Vogel, E. K., \& Luck, S. J. (1998). Sensory gain control (amplification) as a mechanism of selective attention: Electrophysiological and neuroimaging evidence. Philosophical Transcripts of the Royal Society of London, Series B, Biological Sciences, 353, 1257-1270.

Hodsoll, J. P., \& Humphreys, G. W. (2001). Driving attention with the top down: The relative contribution of target templates to the linear separability effect in the size dimension. Perception \& Psychophysics, 63, 918-926.

Hodsoll, J. P., \& Humphreys, G. W. (2005). The effect of target foreknowledge on visual search for categorically separable orientation targets. Vision Research, 45, 2346-2351.

Hopfinger, J. B., Luck, S. J., \& Hillyard, S. A. (2004). Selective attention: Electrophysiological and neuromagnetic studies. In M. S. Gazzaniga (Ed.), The cognitive neurosciences III (3rd ed., pp. 561-574). Cambridge, MA: MIT Press.

Kiss, M., Goolsby, B. A., Raymond, J. E., Shapiro, K. L., Silvert, L., Nobre, A. C., et al. (2007). Efficient attentional selection predicts distractor devaluation: Event-related potential evidence for a direct link between attention and emotion. Journal of Cognitive Neuroscience, 19, 1316-1322.

Kiss, M., Van Velzen, J., \& Eimer, M. (2008). The N2pc component and its links to attention shifts and spatially selective visual processing. Psychophysiology, 45, 240-249.

Kok, A. (2001). On the utility of P3 amplitude as a measure of processing capacity. Psychophysiology, 38, $557-577$.

Lorenzo-Lopez, L., Amenedo, E., \& Cadaveira, F. (2008). Feature processing during visual search in normal ageing: Electrophysiological evidence. Neurobiology of Ageing, 29, 1101-1110.

Luck, S. J., Girelli, M., McDermott, M. T., \& Ford, M. A. (1997). Bridging the gap between monkey neurophysiology and human perception: An ambiguity resolution theory of visual selective attention. Cognitive Psychology, 33, 64-87.

Luck, S. J., \& Hillyard, S. A. (1990). Electrophysiological evidence for parallel and serial processing during visual-search. Perception \& Psychophysics, 48, 603-617.

Luck, S. J., \& Hillyard, S. A. (1994). Spatial-filtering during visual-search-Evidence from human electrophysiology. Journal of Experimental Psychology: Human Perception and Performance, 20, 1000-1014

Luck, S. J., \& Hillyard, S. A. (1995). The role of attention in feature detection and conjunction discrimination: An electrophysiological analysis. International Journal of Neuroscience, 80, 281-297.

Moores, E., Laiti, L., \& Chelazzi, L. (2003). Associative knowledge controls deployment of visual selective attention. Nature Neuroscience, 6, 182-189.

Nieuwenhuis, S., Aston-Jones, G., \& Cohen, J. D. (2005). Decision making, the P3, and the locus coeruleusnorepinephrine system. Psychological Bulletin, 131, 510-532.

Oostenveld, R., \& Praamstra, P. (2001). The five percent electrode system for high-resolution EEG and ERP measurements. Clinical Neurophysiology, 112, 713-719.

Snodgrass, J. G., \& Vanderwart, M. (1980). A standardized set of 260 pictures: Norms and for name agreement, image 
agreement, familiarity, and visual complexity. Journal of Experimental Psychology: Human Learning \& Memory, 6, 174-215.

Soto, D., \& Humphreys, G. W. (2007). Automatic guidance of visual attention from verbal working memory. Journal of Experimental Psychology: Human Perception and Performance, 33, 730-737.

Thompson, K. G., Hanes, D. P., Bichot, N. P., \& Schall, J. D. (1996). Perceptual and motor processing stages identified in the activity of macaque frontal eye field neurons during visual search. Journal of Neurophysiology, 76, 4040-4055.

Wolber, M., \& Wascher, E. (2003). Visual search strategies are indexed by event-related lateralizations of the EEG. Biological Psychology, 63, 79-100.

Woodman, G. F., \& Luck, S. J. (2003). Serial deployment of attention during visual search. Journal of Experimental Psychology: Human Perception and Performance, 29, 121-138. 